\title{
Monitorização Ambulatorial da Pressão Arterial em Indivíduos Normotensos Submetidos a Duas Sessões Únicas de Exercícios: Resistido e Aeróbio
}

\author{
Ambrosina Maria Lignani de Miranda Bermudes, Dalton Valentim Vassallo, Elisardo Corral Vasquez, \\ Eliudem Galvão Lima
}

Vitória, ES

Objetivo - Investigar a influência de duas sessões únicas de exercício resistido (circuito com pesos) e aeróbio sobre as alterações pressóricas, em indivíduos sedentários e normotensos.

Métodos - Foram avaliados pela monitorização numa situação controle, sem realização de exercícios (MAPA 1) 25 indivíduos, após exercício resistido (MAPA 2) e após exercício aeróbio (MAPA 3). Os exercícios resistidos foram realizados sobforma de circuito com pesos, com intensidade de $40 \%$ da força máxima individual e os exercícios aeróbicos em cicloergômetro, com intensidade entre $60 \%$ e $70 \%$ da freqüência cardíaca $(F C)$ máxima alcançada no teste ergométrico.

Resultados - A pressão arterial sistólica (PAS) de $24 \mathrm{~h}$ e sub-períodos vigília e sono não apresentaram variações estatisticamente significantes quando comparada à MAPA2 e MAPA3 e MAPA2 e MAPA3 entre si. A pressão arterial diastólica $(P A D)$ de 24 h e diurna apresentaram reduções significantes $(P<0,05)$. A média da freqüência cardíaca de 24 h e no período vigília apresentou aumentos significativos $(P<0,05)$ quando comparada a MAPA2 à MAPA3.

Conclusão - Uma sessão única de exercício resistido em indivíduos normotensos foi suficiente para promover reduções significativas dos níveis tensionais, no período de sono após o exercício, e a de exercício aeróbio nesses mesmos indivíduos, foi mais eficaz em promover reduções significativas dos níveis pressóricos.

Palavras-chave: MAPA, exercícios resistidos, exercícios aeróbicos

Universidade Federal do Espírito Santo - Escola Superior de Ciências da Santa Casa de Misericórdia de Vitória - EMESCAM

Correspondência: Eliudem Galvão Lima - Rua Guilherme Serrano, 265/ 501

Cep 29055-550 - Vitória, ES - E-mail: eliudem@terra.com.br

Recebido para publicação em 23/10/02

Aceito em 10/3/03
Os diversos estudos que abordam a relação da pressão arterial (PA) com o exercício físico têm se concentrado, principalmente, no exercício do tipo aeróbio dinâmico ${ }^{1-9}$, ou seja, exercícios de natureza contínua, que demandam um período de tempo prolongado e envolvem, na sua execução, grandes grupos musculares. Embora os exercícios aeróbios sejam os mais recomendados e tenham conquistado destaque, quando se fala em promoção de saúde, e atendam bem a esses princípios, ênfase cada vez maior vem sendo dada com o mesmo objetivo à prática de exercícios contra resistência ${ }^{10,11}$. O exercício contra resistência, ou resistido, consiste num trabalho muscular local, que utiliza sobrecargas, como peso de máquinas, barras, anilhas, realizado com cargas moderadas e frequentes repetições, apresentando pausas entre as execuções, e, portanto, caracterizado como esforço descontínuo.

Estudiosos têm se empenhado em esclarecer melhor a importância deste tipo de exercício nas variações pressóricas $^{12-18}$. A qualidade física envolvida neste tipo de esforço físico é a força muscular que, além de ser necessária no desenvolvimento de atividades atléticas, é, em termos de promoção de saúde, um parâmetro essencial para a prática de atividades ocupacionais e de lazer, contribuindo para a auto-suficiência de indivíduos sedentários, idosos, hipertensos e cardiopatas ${ }^{17-19}$. Este tipo de exercício vem sendo, atualmente, utilizado em programas de reabilitação cardíaca, promovendo, quando realizados sob supervisão adequada, benefícios significantes e baixos riscos ${ }^{10}$, e contribuindo para a redução da pressão arterial de repouso. Em uma metaanálise envolvendo indivíduos normotensos e hiperten$\operatorname{sos}^{11}$, observou-se que o exercício resistido dinâmico reduziu em média 3\% a pressão arterial sistólica (PAS) e $4 \%$ a pressão arterial diastólica (PAD) em ambos os grupos, sem que houvesse alteração do peso corporal e da freqüência cardíaca (FC) de repouso. Contudo, o simples fato do exercício resistido leve a moderado não provocar elevações crônicas nos valores pressóricos, já é por si um dado importante, já que as qualidades físicas força e/ou resistência muscu- 
lar localizada são essenciais no desenvolvimento de atividades de rotina, que justificariam a aplicação deste tipo de exercício para efeito de melhora na aptidão física.

Estudos têm demonstrado que o exercício físico agudo (realização de uma única sessão de exercício) é suficiente para provocar uma diminuição na pressão arterial durante o período de recuperação do exercício, tanto em indivíduos normotensos como em hipertensos ${ }^{20-38}$. Os efeitos agudos ocorrem nos períodos pré e imediatamente após o exercício físico. Os efeitos tardios são observados ao longo das primeiras 24h que se seguem a uma sessão de exercícios e podem ser identificados na discreta redução dos níveis tensionais, especialmente nos hipertensos. significando que os níveis pressóricos observados no período de recuperação de exercícios são inferiores àqueles observados no período pré-exercício, ou mesmo daqueles observados em um dia controle sem a execução de exercícios físicos ${ }^{24-35,37,39}$. Essa redução da pressão arterial (PAS e/ou PAD) para valores abaixo dos níveis controle (pré-exercício), após o exercício físico agudo, é denominada redução pressórica pós exercício. Alguns fatores como nível inicial da pressão arterial, tipo e duração do exercício podem influenciar a magnitude e o tempo de duração da redução pressórica. Para que esta redução tenha importância clínica é necessária que ela possua magnitude significativa e perdure por um longo período pós-exercício ${ }^{40}$.

Em recente trabalho ${ }^{41}$, confirmou-se a relevância clínica do exercício agudo, uma vez que a queda dos níveis pressóricos perdurou por $24 \mathrm{~h}$, após uma sessão de exercício físico aeróbio. Também foi demonstrado que a queda da pressão arterial parece ser independente da intensidade do exercício.

As respostas agudas da pressão arterial desencadeadas pelo exercício físico por até $90 \mathrm{~min}$, logo após a prática de uma sessão de exercícios, vêm sendo estudadas, por medida convencional ${ }^{25,26,28,30,37,38}$ ou durante as 24 h após a sessão, através da monitorização contínua da pressão arte rial $^{34,36}$. A maioria dos estudos até agora realizados, baseiase em medidas da pressão arterial em repouso mediante registro casual, as quais, já se sabe, podem sofrer influências de diversas variáveis, como ritmos circadianos, atividades físicas e mentais ${ }^{42,43}$.

Um avanço metodológico a ser considerado neste estudo consiste na utilização da monitorização ambulatorial da pressão arterial (MAPA) para avaliar os efeitos agudos dos exercícios resistido e aeróbio sobre a pressão arterial e a freqüência cardíaca. Avaliamos os poucos trabalhos na literatura em que a MAPA é utilizada após sessão única de exercício resistido e/ou aeróbio e seus resultados são conflitantes. Neste trabalho, investigamos a influência de duas sessões únicas de exercício resistido (circuito com pesos) e aeróbio sobre as alterações pressóricas arteriais e de frequência cardíaca, de um grupo de indivíduos sedentários e normotensos, utilizando-se, a técnica da MAPA e comparando-as entre os dois tipos de exercícios.

\section{Métodos}

Foram estudados 25 indivíduos, entre 40 e 50 anos (média 44ะ1), do sexo masculino, sedentários, não fumantes, assintomáticos, normotensos (PAS < 140mmHg e PAD
<90). O protocolo do estudo foi aprovado pelo comitê de Ética Médica do Centro Biomédico da Universidade Federal do Espirito Santo e os indivíduos apresentaram consentimento por escrito. Em todos os indivíduos foram feitas avaliações do peso, altura e índice de massa corporal (IMC= peso corporal/altura ${ }^{2}$ ).

Antes do inicio do estudo, todos os voluntários participaram de um trabalho de adaptação aos exercícios que seriam executados, a esclarecimentos sobre a técnica de monitorização e ao ambiente do laboratório (semana adaptativa). Após essa semana, todos foram submetidos a teste ergométrico e teste de avaliação da força máxima isotônica, em aparelhagem própria. Posteriormente, os indivíduos foram monitorizados numa situação controle (sem realização de exercícios), após uma sessão de exercício resistido e após uma sessão de exercício aeróbio, definidas aleatoriamente através de sorteio. A monitorização inicial (MAPA1) foi realizada após os indivíduos encontrarem-se em repouso sentado, por $5 \mathrm{~min}$, e no dia não executaram exercícios. A MAPA2 foi realizada pós-exercício resistido e MAPA3 pósexercício aeróbio. O período de intervalo entre MAPA1 e MAPA 2 foi de $48 \mathrm{~h}$, e igual tempo entre MAPA2 e MAPA3. O monitor foi instalado nos indivíduos em torno de 20min após o término do exercício, e mantido por $24 \mathrm{~h}$.

Os testes ergométricos foram realizados em esteira rolante (modelo KT-10200,Inbramed), conjuntamente com registros de eletrocardiograma em repouso e pré esforço nas 12 derivações convencionais, e registros eletrocardiográficos nas derivações MC5, V2 e D2 modificado, nas posições deitado e em pé, em inspiração profunda e após $15 \mathrm{~s}$ de hiperpnéia. O sistema de registro contínuo do eletrocardiograma e os valores de frequiência cardíaca foram acompanhados através de monitor de 3 canais (modelo SM400, TEB). Os testes foram do tipo contínuo (protocolo de Bruce). A pressão arterial e a freqüência cardíaca foram medidas na condição de repouso (deitado e em pé) e ao final de cada estágio. Durante a recuperação, foram medidas até 4 min pós-esforço. Os testes foram realizados até que a exaustão fosse alcançada e a frequiência cardíaca máxima considerada foi a atingida no último estágio do teste. Apenas indivíduos com teste normal ${ }^{44}$ foram incluídos nos protocolos do estudo.

Todos os indivíduos foram submetidos a MAPA, usando um monitor SpaceLabs (modelo 90207), que utiliza a técnica oscilométrica para mensurações da pressão arterial, permitindo gravação automática/manual da pressão arterial e da frequiência cardíaca durante $24 \mathrm{~h}$. O aparelho foi programado para obter as medidas de $15 \mathrm{em} 15 \mathrm{~min}$ no período de 06:00 às 22:00h e a cada 60min de 22:00 às 06:00h da manhã seguinte. A monitorização foi iniciada no dia controle e nos dias pós-exercícios no início da manhã. Considerando que nem todos colocavam o monitor exatamente no mesmo horário optamos, fundamentados na literatura ${ }^{45,46}$, por analisar a curva de 0 a $24 \mathrm{~h}$, considerando o tempo 0 como o momento da colocação do monitor. Os registros eram considerados válidos para interpretação, quando obtínhamos $80 \%$ ou mais de medidas válidas. Os indivíduos foram solicitados a 
manter suas atividades diárias habituais durante o período de leituras e orientados a manter, desde que possível, o membro superior não dominante contendo o manguito, em posição solta e relaxada durante cada medida. Foi fornecido diário para preenchimento detalhado das atividades diárias (sono, trabalho, lazer, alimentação, entre outros). Uma vez que o programa específico do monitor da MAPA apresenta uma programação restrita, em relação aos horários do ciclo sono-vigília e considerando que cada indivíduo tem seu próprio padrão sono/vigília, as médias da pressão arterial e da frequiência cardíaca foram calculadas, a partir dos horários fornecidos por cada um dos indivíduos no preenchimento do seu diário. As normas para interpretação dos resultados foram definidas pelo III Consenso Brasileiro para uso da Monitorização Ambulatorial da Pressão Arterial ${ }^{47}$. Relatório final foi obtido através do software da SpaceLabs.

A força máxima foi avaliada utilizando-se exercícios que envolviam os grupos musculares: grande dorsal, grande peitoral, bíceps braquial, tríceps braquial, bíceps femoral e quadríceps femoral, a fim de se encontrar uma carga máxima na qual o indivíduo só conseguisse executar uma repetição. Foi permitido um número máximo de 3 tentativas para cada exercício. Não foi realizado o teste de carga máxima para os exercícios: glúteo direito e esquerdo, abdominal e dorsallombar. Os exercícios utilizados neste teste foram os mesmos do circuito com pesos a ser implementado, que correspondiam aos mesmos grupos musculares avaliados. Antes da aplicação deste teste os indivíduos foram familiarizados com os equipamentos e protocolos dos exercícios, permitindo sua adaptação, à execução dos movimentos, objetivando excluir a influência destas variáveis no teste e permitir um resultado mais adequado, tipo de avaliação para prescrever a intensidade do trabalho de força, também utilizado por outros autores ${ }^{13,14,17}$.

Os indivíduos foram submetidos a duas sessões únicas de exercícios: uma de exercícios em circuito com pesos (resistido), consistindo 3 séries completas de 10 exercícios (estações) cada, com 20 a 25 repetições (em média 23), realizadas em ritmo moderado e contínuo com intensidade estimada de $40 \%$ da carga máxima (1RM), cada exercício durando em média $45 \mathrm{~s}$, com 30 s de intervalo entre cada um e $2 \mathrm{~min}$ de intervalo entre cada série, e uma sessão de exercício aeróbio, realizada em cicloergômetro (Biocycle Magnetic 2500 , Moviment), numa intensidade entre $60 \%$ a $80 \%$ da freqüência cardíaca máxima alcançada no teste ergométrico, com velocidade entre 60 a 65 rpm e durante 45 min de atividade contínua; precedida por aquecimento na bicicleta sem qualquer carga durante $5 \mathrm{~min}$ e repouso sentado por igual período na recuperação. Exercícios de aquecimento e relaxamento foram realizados antes e depois de cada sessão respectivamente.

Para comparação de duas médias, isto é, a comparação do valor antes e após determinado exercício no mesmo grupo, foi utilizado o teste $t$ de Student para amostras pareadas e para análise das variações horárias da MAPA num mesmo grupo, a análise de variância (ANOVA) para medidas repetidas, de 1-via, seguida de teste de Tukey para identificar os pontos de significância na curva. Para análise das diferen- ças nos valores das curvas temporais entre os 3 grupos estudados, foi utilizada a ANOVA para medidas repetidas, de 2-vias, seguida de teste de Tukey. Os resultados foram expressos em média \pm erro padrão da média (EPM) e nível de significância foi considerado para $\mathrm{P}<0,05$.

\section{Resultados}

Os dados antropométricos, etários e cardiovasculares são apresentados na tabela 1 , onde podemos observar que os indivíduos estudados apresentavam características similares e eram normotensos em repouso.

Os valores da força máxima isotônica realizada no teste de avaliação da carga máxima (teste de uma repetição máxima-1RM) e a intensidade de $40 \%$ da carga máxima (1RM) executada no circuito com pesos, como também os diversos tipos de exercícios utilizados para obtenção de carga máxima e carga de trabalho estão listados na tabela II.

Durante a sessão única de exercícios resistido e aeróbio foi realizada monitorização da frequiência cardíaca com monitor POLAR (modelo Accurex) a fim de se quantificar a intensidade do exercício. Esta medida nos permitiu verificar que os indivíduos do grupo se exercitaram a uma intensida-

\begin{tabular}{|lc|}
\hline \multicolumn{2}{|c|}{ Tabela I - Características antropométricas, etárias, metabólicas e } \\
cardiovasculares
\end{tabular}

\begin{tabular}{|lrr|}
\hline $\begin{array}{c}\text { Tabela II - Tipos de exercícios, dados da carga máxima e da carga de } \\
\text { trabalho executada no exercício em circuito com pesos (resistido) }\end{array}$ \\
\hline Exercícios & Carga Máxima $(\mathrm{Kg})$ & Carga de Trabalho $(\mathrm{Kg})$ \\
\hline Voador dorsal & $25 \pm 1,1$ & $10 \pm 0,5$ \\
Leg press & $142 \pm 5,5$ & $57 \pm 2,2$ \\
Rosca bíceps & $32 \pm 0,8$ & $13 \pm 0,3$ \\
Mesa flexora & $21 \pm 0,8$ & $8,4 \pm 0,3$ \\
Supino plano & $34 \pm 1,4$ & $14 \pm 0,5$ \\
Tríceps pulley alto & $26 \pm 0,6$ & $10,4 \pm 0,2$ \\
\hline Valores expressos em média \pm EPM. \\
\hline
\end{tabular}


de média de $68 \%$ e $65 \%$ da freqüência cardíaca máxima obtida no teste ergométrico, na sessão de exercícios em circuito com pesos (resistido) e na sessão de exercício aeróbio, respectivamente. As variações da frequiência cardíaca durante as sessões únicas de exercícios ficaram dentre dos valores prescritos, ou seja, $60 \%$ a $80 \%$ da frequiência cardíaca máxima obtida no teste ergométrico, segundo orientação do Colégio Americano de Medicina Esportiva ${ }^{48}$.

As medidas da pressão arterial ambulatorial apresentaram boa qualidade, com $95 \%$ de sucesso nas medidas e um total de 69 medidas válidas. Na tabela III encontram-se as médias da pressão arterial e da frequiência cardíaca obtidas nas $24 \mathrm{~h}$, e nos períodos de vigília e sono. Os valores obtidos para as pressões de $24 \mathrm{~h}$, vigília e sono foram normais antes e após os exercícios. O exercício resistido promoveu elevação discreta porém significativa de freqüência cardíaca de 24 h na vigília e elevação da pressão arterial no sono, enquanto que o exercício aeróbio promoveu redução significativa das pressões na MAPA sem alterações de freqüência cardíaca.

As figuras 1, 2 e 3 sintetizam as médias da PAS, PAD e freqüência cardíaca, respectivamente. As variações da PAS (figs. 1a e 1b) não foram estatisticamente significantes. As médias da PAD (figs. 2a e 2b) apresentaram reduções significativas $(\mathrm{P}<0,05)$ quando comparadas as medidas do controle com as medidas pós-exercício aeróbio. No período de sono apresentaram reduções de $5 \%$ e $5,3 \%$ ( $\mathrm{P}<0,01)$ respectivamente quando comparadas as medidas controle com as medidas pós-exercício resistido e controle com medidas pós-exercício aeróbio.

As médias da frequiência cardíaca (figs. 3a e 3b) apresentaram nas $24 \mathrm{~h}$ e no período de vigília aumentos significativos de $2,5 \%(\mathrm{P}<0,05)$ quando comparadas as medidas pós-exercício resistido com as pós-exercício aeróbio.

\begin{tabular}{|c|c|c|c|}
\hline \multicolumn{4}{|c|}{$\begin{array}{l}\text { Tabela III - Dados da pressão arterial (PA) e da frequência cardíaca } \\
\text { (FC) obtidos na monitorização ambulatorial da pressão arterial } \\
\text { (MAPA) controle, pós-exercício resistido e pós-exercício aeróbio }\end{array}$} \\
\hline Parâmetros & Controle & Resistido & Aeróbio \\
\hline $\mathrm{N}$ & 25 & 25 & 25 \\
\hline \multicolumn{4}{|l|}{ Média 24 h } \\
\hline PAS (mm Hg) & $121 \pm 1,3$ & $121 \pm 1,3$ & $121 \pm 1,3$ \\
\hline $\mathrm{PAD}(\mathrm{mm} \mathrm{Hg})$ & $79 \pm 1$ & $78,3 \pm 1$ & $77,6 \pm 1,0$ \\
\hline $\mathrm{FC}(\mathrm{bpm})$ & $77 \pm 1,8$ & $78,6 \pm 2 \otimes$ & $76,5 \pm 1,7$ \\
\hline \multicolumn{4}{|l|}{ Média vigília } \\
\hline PAS (mm Hg) & $123 \pm 1,4$ & $123 \pm 1,4$ & $122 \pm 1,3$ \\
\hline $\mathrm{PAD}(\mathrm{mm} \mathrm{Hg})$ & $80,6 \pm 1$ & $79,6 \pm 1,1$ & $78,9 \pm 1,1$ \\
\hline $\mathrm{FC}(\mathrm{bpm})$ & $78,4 \pm 1,9$ & $80 \pm 2,1 \otimes$ & $77,6 \pm 1,7$ \\
\hline \multicolumn{4}{|l|}{ Média sono } \\
\hline PAS (mm Hg) & $107 \pm 1,1$ & $106 \pm 1,1$ & $105 \pm 1,3$ \\
\hline $\mathrm{PAD}(\mathrm{mm} \mathrm{Hg})$ & $66,4 \pm 0,8$ & $63 \pm 0,9 * *$ & $62,9 \pm 1,1 \bullet \bullet$ \\
\hline FC (bpm) & $64,7 \pm 1,4$ & $64,6 \pm 1,7$ & $63,3 \pm 1,4$ \\
\hline \multicolumn{4}{|c|}{$\begin{array}{l}\text { Valores expressos em média } \pm \text { EPM. PAS - pressão arterial sistólica; PAD - } \\
\text { pressão arterial diastólica, valores em mmHg). } * * \mathrm{P}<0,01 \text { indicando significância } \\
\text { (MAPA controle vs. MAPA pós-exercício resistido). } \bullet \mathrm{P}<0,05 \text { e } \bullet \mathrm{P}<0,01 \\
\text { indicando significância (MAPA controle vs. MAPA pós-exercício aeróbio) e } \\
\otimes \mathrm{P}<0,05 \text { indicando significância (MAPA pós-exercício resistido vs. MAPA } \\
\text { pós-exercício aeróbio). - Teste } t \text { para amostras pareadas. }\end{array}$} \\
\hline
\end{tabular}

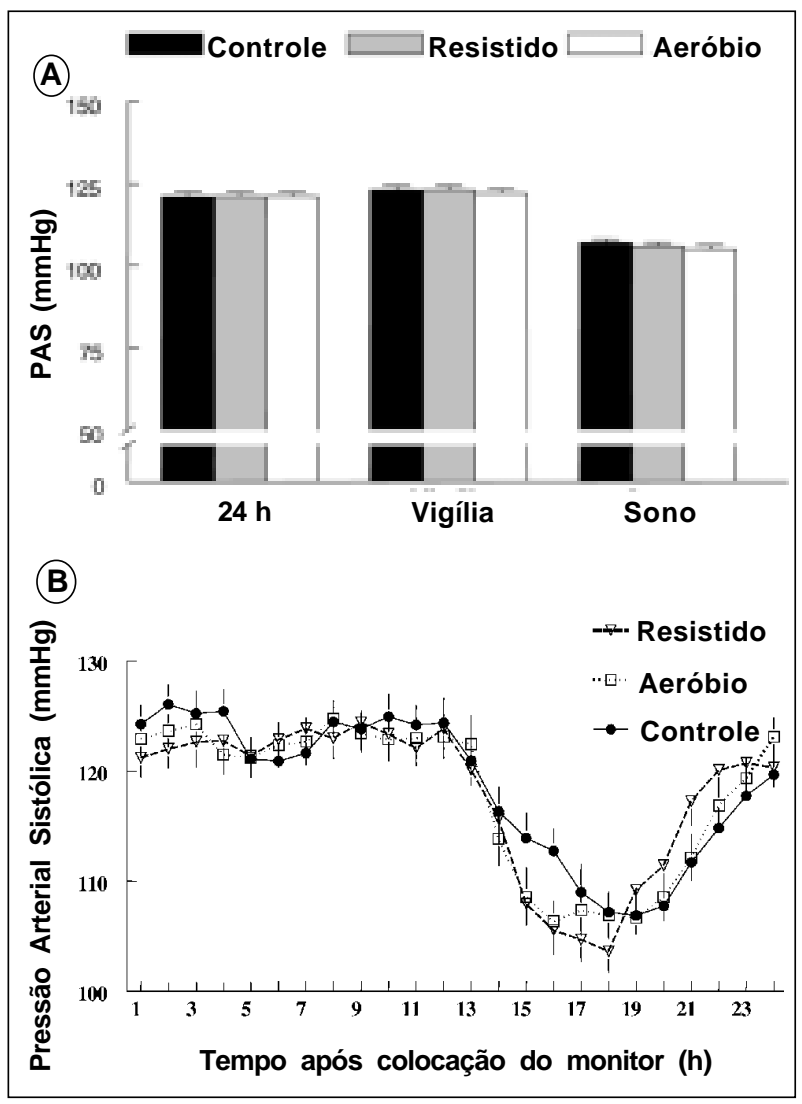

Fig. 1: A - Médias da PAS obtidas na MAPA. MAPA controle $v$ s. MAPA pós-exercício resistido; MAPA controle $v$ s. MAPA pós-exercício aeróbio e MAPA pós-exercício resistido $v$ s. MAPA pós-exercício aeróbio; B - Evolução temporal dos valores de pressão arterial sistólica após a colocação do monitor da MAPA. As diferenças foram significantes quando comparado controle $v$ s. resistido e controle $v$ s. aeróbio nos tempos 15 e $16 \mathrm{~h}$. Entre o resistido e aeróbio houve diferença significante no tempo de $21 \mathrm{~h}$.

\section{Discussão}

Grande parte dos estudos $20-23,27,30-33,35-39$ têm demonstrado que o exercício físico agudo aeróbio e resistido provoca queda pressórica duradoura no período de recuperação pós exercício, isto é, provoca uma redução pressórica pósexercício. Parece claro que a magnitude e a duração da queda pressórica podem ser influenciadas por diversos fatores, como a amostra estudada (normotensos ou hipertensos), o tipo, a intensidade e a duração do exercício ${ }^{40}$.

Neste estudo, avaliamos, em um mesmo grupo de indivíduos, o efeito do exercício agudo em circuito com pesos (resistido) e do exercício agudo aeróbio, devidamente padronizados, no comportamento da pressão arterial e da freqüência cardíaca durante a realização de atividades habituais; utilizando-se da MAPA, permitindo-nos avaliar o comportamento dessas variáveis hemodinâmicas durante as $24 \mathrm{~h}$ do dia.

Pouca ênfase tem sido dado a parâmetros cardiovasculares após sessão única de exercício resistido ${ }^{49}$. Em relação aos exercícios agudos do tipo aeróbio, alguns estudos $^{24,34,36}$ utilizaram a técnica da MAPA após sessão única de exercício. 


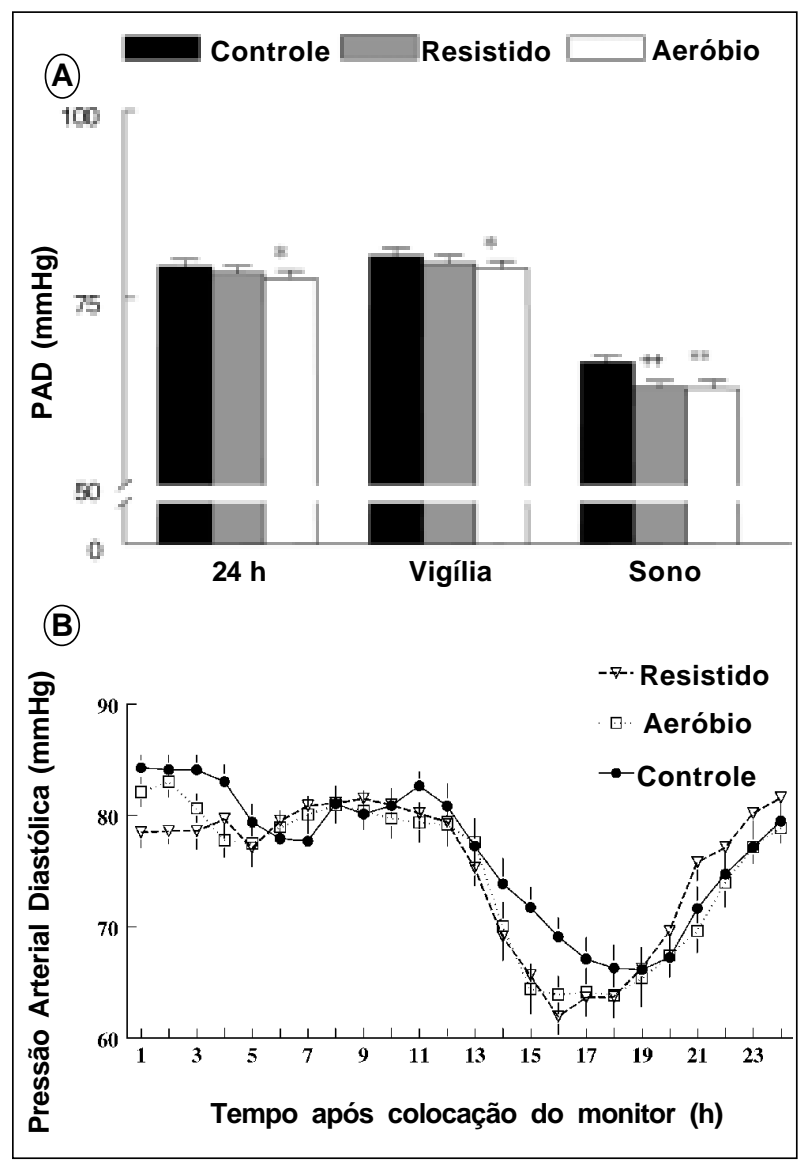

Fig. 2: A - Médias da PAD obtidas na MAPA. ${ }^{++} \mathrm{P}<0,01$ indicando significância MAPA controle $v$ s. MAPA pós-exercício resistido. ${ }^{*} \mathrm{P}<0,05$ e ${ }^{* *} \mathrm{P}<0,01$ indicando significância MAPA controle vs. MAPA pós-exercício aeróbio; B - Evolução temporal dos valores de pressão arterial diastólica após a colocação do monitor da MAPA. As diferenças foram significantes quando comparado controle $v$ s. resistido nos tempos 1, 2, 3, 14, 15 e $16 \mathrm{~h}$ e controle $v$ s. aeróbio nos tempos 4, 15 e $16 \mathrm{~h}$. Entre o resistido e aeróbio houve diferença significante no tempo de 2 e $21 \mathrm{~h}$.

Estudos recentes ${ }^{7,9,48,50}$ enfatizam a MAPA por permitir a execução de medidas pressóricas em intervalos pré-determinados, durante atividades de rotina e do sono, oferecendo uma curva representativa do comportamento pressórico do indivíduo durante $24 \mathrm{~h}$. Portanto, há uma importante distinção entre o presente estudo e grande parte dos trabalhos encontrados na literatura ${ }^{22,25,28,30,37-39}$ : enquanto os demais trabalhos tratam do exercício aeróbico e resistido, individualmente, através da medida convencional ou da MAPA, no nosso estudo os indivíduos foram submetidos a ambos os exercícios, comparados durante o período de $24 \mathrm{~h}$, e avaliados pela MAPA, com registros dos níveis pressóricos durante a execução de suas atividades habituais, na vigília e no sono; procedimento este pouco utilizado pós-esforço ${ }^{24,34,36}$.

Com relação à resposta da PAS após a sessão única de exercício aeróbio, nossos resultados são conflitantes com a literatura, porque a maioria dos estudos ${ }^{20,28,35,36,51}$ encontrou reduções significativas da PAS, variavando de 3 a $9 \mathrm{mmHg}$, em indivíduos normotensos. Entretanto, corroboram os resultados de outros estudos ${ }^{24,26}$ que não observaram queda desse parâmetro em indivíduos normotensos. Pescatello e cols ${ }^{24}$ avaliando indivíduos normotensos e hi-

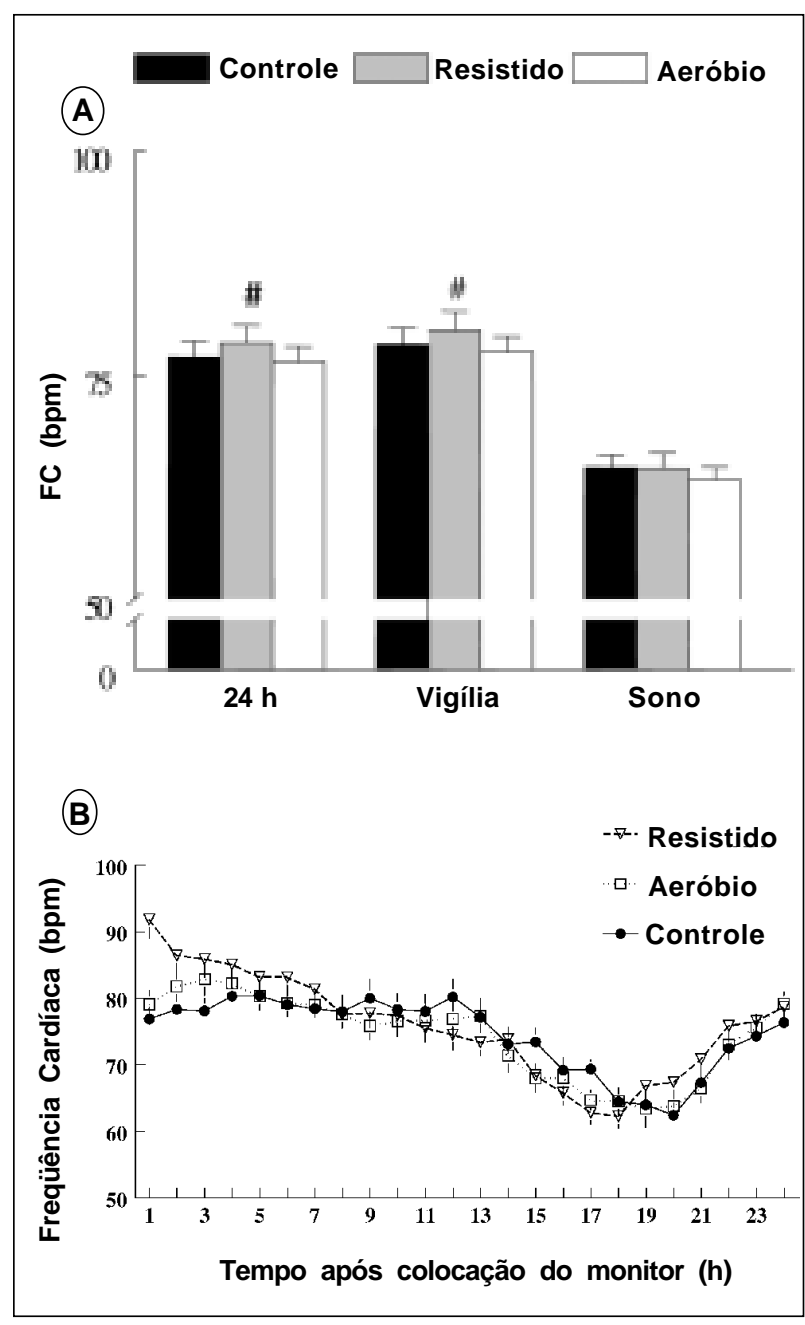

Fig. 3: A - Médias da FC obtidas na MAPA. \#P $<0,05$ indicando significância MAPA pós-exercício resistido $v$ s. MAPA pós-exercício aeróbio; B - Evolução temporal dos valores de frequiência cardíaca após a colocação do monitor da MAPA. As diferenças foram significantes quando comparado controle $v$ s. resistido nos tempos 1,2,3,12 e $17 \mathrm{~h}$ e controle $v$ s. aeróbio nos tempos $15 \mathrm{~h}$. Entre o Resistido e Aeróbio houve diferença significante no tempo de $1 \mathrm{~h}$.

pertensos, mensuraram a resposta da pressão arterial ambulatorial em relação ao exercício aeróbio agudo de intensidades diferentes, durante 13h após o esforço, e não demonstraram reduções da pressão arterial em normotensos, mas sim reduções significativas da PAS $(5 \mathrm{mmHg})$ e da PAD $(8 \mathrm{mmHg})$ nos hipertensos. Em relação às médias dos valores de PAS após a sessão única de exercício resistido, nossos resultados confirmam os de outros autores ${ }^{28,39,47,50}$; que também não verificaram redução significativa desse parâmetro em indivíduos normotensos.

Quanto à resposta da PAD após a sessão única de exercício aeróbio, nossos resultados confirmam os de outros es$\operatorname{tudos}^{30,35,51,52}$, com reduções significativas desse parâmetro entre 2 e $6 \mathrm{mmHg}$, porém são contrários aos resultados de outros pesquisadores ${ }^{20,28}$, cujas reduções significativas da PAD em indivíduos normotensos não foram encontradas. Nossos resultados também corroboram outro estudo ${ }^{36}$, em que queda significativa da PAD $(2 \mathrm{mmHg})$ após exercício agudo aeróbio, persistindo por $24 \mathrm{~h}$, parece ser predominan- 
te no período do sono. Entretanto, com relação ao período de vigília, esse mesmo estudo não encontrou variações significativas da PAD, enquanto em nosso estudo foi achada redução significativa da PAD no mesmo período. Tratando-se de PAD, mesmo as reduções, por nós encontradas nas $24 \mathrm{~h}$, e períodos de vigília e sono, acreditamos que tenha significado clínico relevante.

Hill e cols. ${ }^{51}$, estudando indivíduos normotensos com idade entre 22 e 33 anos, após realização de exercício resistido com intensidade de 70\% 1 RM e duração média de 14min, relataram queda significativa $(8 \mathrm{mmHg})$ na PAD e não significativa, após uma sessão de resistência muscular de baixa intensidade, observada por Brown e cols ${ }^{33}$. Outros autores ${ }^{39}$ constataram, que após $50 \%$ de carga voluntária máxima, a PAD caiu significativamente. Em nosso estudo, encontramos redução da PAD durante o sono pós-exercício resistido, resultado que vai de encontro ao observado em outro estudo ${ }^{28}$, que concluiu não haver queda da pressão arterial após sessão única de exercício resistido.

Estudos realizados com indivíduos normotensos ${ }^{24} \mathrm{ob}-$ servaram valores menores de pressão arterial antes do exercício (controle) naqueles indivíduos, e que a sua diferença absoluta na recuperação do exercício é bem menor que a encontrada nos indivíduos hipertensos, e, portanto, menos provável de alcançar significância estatística.

O comportamento da frequiência cardíaca durante o período de recuperação do exercício tem apresentado resultados controversos na literatura. Rueckert e cols. ${ }^{34}$ estudaram indivíduos hipertensos, de ambos os sexos, e observaram que após sessão de exercício aeróbio, a freqüência cardíaca estava significativamente aumentada nas $3 \mathrm{~h}$ seguintes ao exercício. Esses autores sugeriram que essa taquicardia, mantida na recuperação do exercício, podia ser motivada pelo mecanismo barorreflexo influenciado pela queda da pressão arterial ou uma redução pós-exercício da atividade vagal com exacerbação da atividade simpática para o coração, segundo outros autores ${ }^{30}$. Outros estudos não relataram alterações da freqüência cardíaca ${ }^{21,22,24,36}$. Suas variações, após o exercício físico, também foram observadas ${ }^{38} \mathrm{e}$ as diferenças parecem estar relacionadas à intensidade do exercício, pois os autores verificaram que, após 45min de exercício aeróbio leve (30\% do consumo máximo de oxigênio), observava-se sua queda, após exercício moderado (50\% do consumo máximo de oxigênio), seu aumento e, após exercício mais intenso (80\% do consumo máximo de oxigênio), seu aumento transitório.

Vários trabalhos na literatura ${ }^{53-58}$ consideram a medida da frequiência cardíaca como um parâmetro a mais na análise do comportamento das variáveis cardiovasculares obtidas através da monitorização ambulatorial das 24 h e o analisando, encontramos em nossos resultados aumentos significativos $(2,5 \%)$ na média de 24 horas e no período de vigília, quando comparamos a MAPA pós-exercício resistido com a MAPA pós-exercício aeróbio, ao passo que comparando a monitorização controle com a MAPA pós-exercício aeróbio e pós-exercício resistido, não encontramos diferenças estatisticamente significativas. Nossos resultados corroboram os de outros pesquisadores ${ }^{24}$ que não encontraram em indivíduos normotensos diferenças significativas na freqüência cardíaca antes e após sessão única de exercício aeróbio, o mesmo acontecendo em outro estudo ${ }^{47}$ onde a frequiência cardíaca é significativamente aumentada após sessão única de exercício resistido.

Médias de freqüência cardíaca maiores foram observadas na curva de $24 \mathrm{~h}$, obtida, a cada hora após a colocação do monitor, quando comparamos a MAPA controle com a MAPA pós-exercício resistido. Entretanto, esses aumentos significativos foram isolados e ocorreram nas $3 \mathrm{~h}$ após o exercício. Não houve um deslocamento da curva para cima. Nossos resultados corroboram os de O'Connor e cols. ${ }^{47}$, que relataram que a frequiência cardíaca após sessão única de exercício resistido está significativamente aumentada nas 2 h que seguem o exercício. Não sendo a MAPA o método mais indicado para se aferir freqüência cardíaca, torna-se necessário em estudos futuros, empregar-se outra metodologia para que se tenham dados menos controversos quanto a esse parâmetro.

Vários autores têm procurado explicar os mecanismos envolvidos na redução da pressão arterial no período de recuperação do exercício. É possível que a queda pressórica na recuperação do exercício se deva, principalmente, à diminuição da resistência vascular periférica total ${ }^{25,30,31,34,35}$. Redução que pode estar relacionada à vasodilatação provocada pelo exercício físico, tanto na musculatura ativa ${ }^{34,35}$, como na musculatura inativa ${ }^{25,26,31}$. Piepoli e cols. ${ }^{31}$ relataram que a vasodilatação nos músculos inativos pode estar relacionada à intensidade do exercício. Hagberg e cols. ${ }^{32}$ demonstraram que a redução do débito cardíaco é o mecanismo responsável pela redução pressórica pós-treinamento físico. Através da técnica de reinalação e equilíbrio de $\mathrm{CO}_{2}$, esses autores verificaram que a queda de pressão arterial após um período de treinamento físico estava associada à redução do débito cardíaco em decorrência de uma bradicardia de repouso, uma vez que não foram observadas alterações significativas no volume sistólico ${ }^{32}$. Em recente estudo, Rondon e cols. ${ }^{59}$ observaram, em idosos hipertensos não obesos, que a redução pressórica pós-exercício estava associada à queda do volume diastólico final do ventrículo esquerdo e, como conseqüência, do volume sistólico e débito cardíaco. Outros mecanismos também têm sido propostos, tais como: mecanismos termo-regulatórios ${ }^{27}$; aumento do fluxo sangüíneo muscular, como provável consequiência da diminuição da atividade simpática periférica ${ }^{25,60}$; modulação direta de opióides endógenos sobre o fluxo sangüíneo, aumentando a vasodilatação periférica ${ }^{61}$; alterações no funcionamento dos barorreceptores arteriais ${ }^{22}$ e receptores cardiopulmonares ${ }^{26} \mathrm{de}$ volume, como aumento na sua sensibilidade e alteração no ponto de ajuste desses reflexos na recuperação do exercício, podendo contribuir para o efeito vasodilatador pós-exercício. Rueckert e cols. ${ }^{34}$ observaram que a redução pressórica pós-exercício era determinada por mecanismo bifásico, ou seja, dependia da queda da resistência vascular periférica nos 30min iniciais da recuperação e da queda do débito cardíaco, após este período. Portanto, os mecanismos responsáveis pela redução pressórica pósexercício ainda são controversos, podendo estar relacionados, além de outros fatores, à redução do débito cardíaco ou da resistência vascular periférica.

Alguns estudos ${ }^{39,62}$ também têm avaliado mecanismos 
relacionados à redução do estresse e da ansiedade pósexercício, sugerindo que o efeito ansiolítico, tanto do exercício aeróbio quanto do exercício resistido, tenha importância na queda pressórica pós-exercício. Ressaltamos, entretanto, que os mecanismos envolvidos na gênese da redução pressórica não foram objeto de nossa investigação.

Nossos resultados comprovaram que uma sessão única de exercício resistido foi eficaz em promover reduções significativas dos níveis tensionais de indivíduos normotensos no período de sono, após o exercício, quando avaliados pela MAPA. Também a sessão única de exercício aeró- bio foi mais eficaz em promover reduções significativas dos níveis pressóricos desses mesmos indivíduos, tendo em vista que ocorreram reduções da pressão arterial nas $24 \mathrm{~h}$, nos períodos vigília/sono, após o exercício. Entretanto, a frequiência cardíaca desses indivíduos encontrou-se elevada nas $24 \mathrm{~h}$ no período de vigília e durante a recuperação do exercício agudo resistido.

Mais esclarecimentos se fazem necessários para que se estabeleça o papel do exercício físico agudo na modulação da pressão arterial, e a contribuição da MAPA, seguramente, representará um avanço para estudos futuros.

\section{Referências}

1. Gilders RM, Voner C, Dudley GA. Endurance training and blood pressure in normotensive and hypertensive adults. Med Sci Sports Exer 1989; 21: 629-36

2. Van Hoof R, Hespel P, Fagard R, et al. Effect of endurance training on blood pressure at rest, during exercise and during 24 hours in sedentary men. Am J Cardiol 1989; 63: 945-9.

3. Kelley G \& McClellan P. Antihypertensive effects of aerobic exercise: a brief metaanalytic review of randomized controlled trials. Am J Hypertens 1994; 7:115-9.

4. Wijnen JAG, Kool MJF, Van Baak MA, et al. Effect of exercise training on ambulatory blood pressure. Int J Sports Med 1994;15: 10-5.

5. Fagard RH. Prescription and results of physical activity. J Cardiovasc Pharmacol 1995; 25: S20-7.

6. Fagard RH. The role of exercise in blood pressure control: supportive evidence. J Hypertens 1995; 13: 1223-7.

7. Lima EG, Spritzer N, Herkenhoff FL, et al. Noninvasive ambulatory 24 hour blood pressure in patients with high normal blood pressure and exaggerated systolic pressure response to exercise. Hypertension 1995;26: 1121-4.

8. Arida RM, Naffah-Mazzacoratti MG, Soares J, et al. Effect of an aerobic exercise program on blood pressure and catecholamines in normotensive and hypertensive subjects. Braz J Med Biol Res 1996; 29: 633-7.

9. Lima EG, Herkenhoff F, Vasquez EC. Monitorização ambulatorial da pressão arterial em indivíduos com resposta exagerada dos níveis pressóricos em esforço. Influência do condicionamento físico. Arq Bras Cardiol 1998;70: 1-7.

10. Verrill DE, Ribisl PM. Resistive exercise training in cardiac rehabilitation: an update. Sports Med 1996;21: 347-83.

11. Kelley G. Dynamic resistence exercise and resting blood pressure in adults: a meta analysis. J Appl Physiol 1997;82: 1559-65.

12. Allen TE, Byrd RJ, Smith DP. Hemodynamic consequences of circuit weight training. Res Quat 1976; 47: 299-306.

13. Gettman LR, Ward P, Hagan RD. A comparison of combined running and weight training with circuit weight training. Med Sci Sports Exer 1982;16:207-15.

14. Harris KA, Holly RG. Physiological response to circuit weight training in borderline hypertensive subjects. Med Sci Sports Exer 1987;19: 246-52.

15. Fleck SJ. Cardiovascular adaptations to resistence training. Med Sci Sports Exer 1988;20: S146-51.

16. Hurley BF, Hagberg JM, Goldberg AP, et al. Resistive training can reduce coronary risk factors without altering $\mathrm{VO}_{2}$ max or percent body fat. Med Sci Sports Exer 1988;20:150-4

17. Kelemen MH. Resistive training safety and assessment guidelines for cardiac and coronary prones patients. Med Sci Sports Exer 1989;21: 675-7.

18. Stewart KJ. Weight training in coronary artery disease and hypertension. Prog Cardiovasc Dis 1992;35: 159-68.

19. Morrissey MC, Harman EA, Johnson MJ. Resistence training modes: specificity and effectiveness. Med Sci Sports Exer 1995;27: 648-60.

20. Hannum SM, Kasch FW. Acute postexercise blood pressure response of hypertensive and normotensive men. Scand J Sports Sci 1981;3:11-5.

21. Wilcox RG, Bennett T, Brown AM et al. Is exercise good for high blood pressure? Braz Med J 1982;285: 767-9.

22. Bennet T, Wilcox RG, MacDonald IA. Post-exercise reduction of blood pressure in hypertensive men is not due to acute impairment of baroreflex function. Clin Sci 1984;67:97-103.

23. Kaufman FL, Hughson RL, Schaman JP. Effect of exercise on recovery blood pressure in normotensive and hypertensive subjects. Med Sci Sports Exer 1987; 19:17-20.
24. Pescatello LS, Fargo AE, Jr Leach CN et al. Short-term effect of dynamic exercise on arterial blood pressure. Circulation 1991;83: 1557-61.

25. Cléroux J, Kouamé N, Nadeau A, et al. After effects of exercise on regional and systemic hemodinamics in hypertension. Hypertension 1992;19: 183-91.

26. Cléroux J, Kouamé N, Nadeau A, et al. Barroreflex regulation of forearm vascular resistence after exercise in hypertensive and normotensive humans. Am J Physiol 1992b;263: H1523-31.

27. Franklin PJ, Green DJ, Cable NT. The influence of thermoregulatory mechanisms on post-exercise hypotension in humans. J Physiol 1993;470: 231-41.

28. Raglin JS, Turner PE, Eksten F. State anxiety and blood pressure following $30 \mathrm{mi}-$ nutes of leg ergometry or weight training. Med Sci Sports Exer 1993;25: 1044-8.

29. Boone JB, Probst MM, Rogers MW, et al. Postexercise hypotension reduces cardiovascular responses to stress. J Hypertens 1993; 11: 449-53.

30. Piepoli M, Coats AJS, Adamopoulos S, et al. Persistent peripheral vasodilatation and sympathetic activity in hypotension after maximal exercise. J Appl Physiol 1993; 75: 1807-14

31. Piepoli M, Isea JE, Pannarale G, et al.Load dependence of changes in foerearm and peripheral vascular resistence after acute leg exercise in man. JPhysiol 1994;478: 357-60.

32. Hagberg JM, Montain SJ, Martin WH, et al. Effect of exercise training in 60 to 69 year old persons with essential hypertension. Am J Cardiol 1989;64: 348-53.

33. Brown SP, Clemons JM, He Qin, et al. Effects of resistence exercise and cycling on recovery blood pressure. J Sports Sci 1994;12: 463-8.

34. Rueckert PA, Slane PR, Lillis DL, et al. Hemodynamics patterns and duration of post-dynamic exercise hypotention in hypertensive humans. Med Sci Sports Exer 1996;28:24-32.

35. Halliwill JR, Taylor A, Eckberg DL. Impaired sympathetic vascular regulation in humans after acute dynamic exercise. J Physiol 1996;495: 279-88.

36. Forjaz CLM, Mion Jr. D, Negrão D. The fall in blood pressure following a single bout of endurance exercise is sustained for 24 hours. (Abstract).Hypertension 1995;25: 1400.

37. Forjaz CLM, Matsudaira Y, Rodrigues FB, et al. Post-exercise changes in blood pressure, heart rate and rate pressure product at diferents exercise intensities in normotensive humans. Braz J Med Biol Res 1998; 31:1247-55.

38. ForjazCLM, SantaellaDF, RezendeLO, et al. Aduraçãodoexercíciodeterminaamagnitude e a duração da hipotensão pós-exercício. Arq Bras Cardiol 1998;70: 99-104.

39. Focht BC, Koltyn KF. Influence of resistance exercise of diferents intensities on state anxiety and bloo pressure. Med Sci Sports Exer 1999;3:456-63.

40. Kenney MJ, Seals DR. Post exercise hypotension - key features, mechanisms and clinical significance. Hypertension 1993;22: 653-4.

41. Negrão CE, Rondon MUPB. Exercício físico, hipertensão e controle barorreflexo da pressão arterial. Rev Bras Hipertens 2001; 8:89-95.

42. Kaplan NM. Treatment of hypertension: nondrug therapy. In: Kaplan NM. Clinical Hypertension. Baltimore: Williams \& Wilkins, 1994; 171-89.

43. Krieger EM. Variabilidade da pressão arterial durante a vigília e o sono. In: Jr Mion D, Nobre F, Oigman W (Eds.). Monitorização Ambulatorial da Pressão Arterial, São Paulo: Atheneu, 1994;2:19-29.

44. Ellestad MH. Stress Testing. Principles and Practice. Philadelphia: Davis Company, 1986

45. Fagard R. Exercise and hypertension. J Human Hypertens 1999; 13: 359-60.

46. Prasad N, MacFadyen RJ, Ogston SA, MacDonald TM. Elevated blood pressure during the first two hours of ambulatory blood pressure monitoring: a study comparing consecutive twenty - four - hour monitoring periods. J Hypertens 1995; 13:291-5.

47. III Consenso Brasileiro para o uso da Monitorização Ambulatorial da Pressão Arterial 2001 
48. American College of Sports Medicine Position Stand. The recommended quantity and quality of exercise for developing and mantaining cardiorespiratory and muscular fitness in healthy adults. Med Sci Sports Exer 1990;22: 265-74.

49. O'ConnorPJ, Bryant CX, VeltriJP, et al. State anxiety and ambulatory blood pressure following resistence exercise in females. Med Sci Sports Exer 1993;25: 516-21.

50. Mancia G, Sega R, Bravi C, et al. Ambulatory blood pressure normality: results from the Pamela study. J Hypertens 1995; 13: 1377-90.

51. Hill DW, Collins MA, Cureton KJ, et al. Blood pressure response after weight training exercise. J Appl Sport Sci Res 1989; 3: 44-7

52. Staessen JA, Bieniaszewski L, O'Brien E, et al. Nocturnal blood pressure fall on ambulatory monitoring in a large international data base. Hypertension 1997;29: 30-9.

53. Mengden T, Wesseir B, Vetter W. Ambulatory 24-hour blood pressure versus sefmeasured blood pressure in pharmacological trials. J Cardiovasc Pharmacol 1994;24:S20-5

54. Nami R, MondilloS, AgricolaE, et al. Aerobic exercise training fails to reduce blood pressure in nondipper - type hipertension. Am J Hipertens 2000; 13: 593-600.
55. Taylor-TolberNS, DengelDR, Bown MiD, etal. Ambulatory blood pressure afteracute exercise in older men with essential hypertension. Am J Hypertens 2000; 13: 44-51.

56. Sega R, Cessana G, Costa G, et al. Ambulatory blood pressure in air traffic controllers. Am J Hypertens 1998; 11:208-12.

57. Marceau M,KouaméN,Laucourcière Y,etal.Effects of different training intensities on 24-hour blood pressure in hypertensive subjects. Circulation 1993; 88: 2803-11.

58. Mancia G, Sega R, Bravi C, et al. Ambulatory blood pressure normality: results from the PAMELA study. J Hypertens 1995; 13:1377-90.

59. Rondon MUPB, Alves MJNN, Braga AMFW, et al. Postexercise blood pressure reduction in elderly hypertensive patients. Hypertension 2002;39: 676-82.

60. Floras JS, Sinkey CA, Aylward PE, et al. Post exercise hypotention and sympathoinhibition in boderline hypertensive men. Hypertension 1989;14: 28-35.

61. Thorén P, Floras JS, Hoffmann P, et al. Endorphins and exercise: physiological mechanisms and clinical implications. Med Sci Sports Exer 1990;22: 417-28.

62. Koltyn KF, Raglin JS, O'Connor PJ, et al. Influence of weight training on state anxiety, body awareness and blood pressure. Int J Sports Med 1995;16:266-9. 